\title{
Outline shape is a mediator of object recognition that is particularly important for living things
}

\author{
TOBY J. LLOYD-JONES and LINDA LUCKHURST \\ University of Kent, Canterbury, England
}

\begin{abstract}
We assess the importance of outline shape in mediating the recognition of living and nonliving things. Natural objects were presented as shaded line drawings or silhouettes, and were living and nonliving things. For object decision (deciding whether an object may be encountered in real life) there were longer response times (RTs) to nonliving than to living things. Importantly, this category difference was greater for silhouettes than for shaded line drawings. For naming, similar category and stimulus differences were evident, but were not as pronounced. We also examined effects of prior naming on subsequent object decision performance. Repetition priming was equivalent for nonliving and living things. However, prior presentation of silhouettes (but not shaded line drawings) reduced the longer RT to nonliving things relative to living things in silhouette object decision. We propose that outline contour benefits recognition of living things more than nonliving things: For nonliving things, there may be greater 2-D/3-D interpretational ambiguity, and/or they may possess fewer salient features.
\end{abstract}

It is well established that neurologicallyimpaired individuals may show selective difficulties in the recognition of living things (e.g., Farah, McMullen, \& Meyer, 1991; Riddoch \& Humphreys, 1987; Sartori \& Job, 1988; Sartori, Job, \& Coltheart, 1993; Sheridan \& Humphreys, 1993; Silveri \& Gainotti, 1988; see also a special issue of Neurocase, 1998). In addition, the opposite dissociation of impaired recognition of nonliving things has been reported, albeit less frequently (e.g., Hillis \& Caramazza, 1991; Sacchett \& Humphreys, 1992; Warrington \& McCarthy, 1983, 1987). The simple living-nonliving dichotomy does not capture fully the difference between impaired and unimpaired categories. For instance, deficits for living things, but not for body parts, have been found to co-occur with loss of knowledge about musical instruments (Warrington \& Shallice, 1984). On the basis of this, it has been argued that semantic knowledge is represented in the brain by modality (visual, olfactory, motor/functional, and so on) and that whereas sensory properties are more important for comprehending living things, functional properties are more important for comprehending nonliving things (e.g., Gainotti \& Silveri, 1996; Hart \& Gordon, 1992; Warrington \& McCarthy, 1983, 1987; Warrington \& Shallice, 1984).

For a number of reasons, however, this interpretation remains controversial. First, it is difficult to account for all

This research was supported by an Economic and Social Research Council grant T.J.L.-J. (Grant R000222174). Correspondence should be addressed to T. J. Lloyd-Jones, Department of Psychology, University of Kent, Canterbury, Kent CT1 2NP, United Kingdom (e-mail: t.j.lloydjones@ukc.ac.uk).We thank Colin M. MacLeod, William Hayward, and an anonymous reviewer for comments on an earlier version of this article.

-Accepted by previous editorial team category-specific impairments in terms of selective loss of sensory or functional properties (Lambon-Ralph, Howard, Nightingale, \& Ellis, 1998; Samson, Pillon, \& De Wilde, 1998). Second, deficits may reflect a selective problem in lexical retrieval rather than retrieval of visual or semantic knowledge (Farah \& Wallace, 1992; Hart, Berndt, \& Caramazza, 1985; Luckhurst \& Lloyd-Jones, 2001). Finally, and of most relevance here, authors have argued that category-specific deficits in the recognition and naming of living things may be artefactual (Funnell \& Hodges, 1996; Funnell \& Sheridan, 1992; Lloyd-Jones \& Humphreys, 1997a, 1997b; Stewart, Parkin, \& Hunkin, 1992).

\section{Differences in Normal Object Processing of Living and Nonliving things}

Studies of normal object naming have suggested that differences in response times (RTs) and accuracy to living and nonliving things may arise due to normal processing differences, between objects from categories with many perceptually similar exemplars ("structurally similar" objects such as fruits, vegetables, and animals) and objects from categories with visually more distinctive exemplars ("structurally distinct" objects such as clothing, furniture, and vehicles) (e.g., Humphreys, Riddoch, \& Quinlan, 1988; Lloyd-Jones \& Humphreys, 1997a, 1997b). LloydJones and Humphreys (1997a, 1997b) in particular have argued that living things require greater visual differentiation from perceptual neighbors than do nonliving things.

The studies just outlined used unlimited exposure durations. However, a short exposure duration can eliminate (Humphreys, Lloyd-Jones, \& Fias, 1995) and indeed reverse (Laws \& Neve, 1999) this disadvantage for living relative to nonliving things. ${ }^{1}$ Laws and Neve suggested that poorer accuracy for nonliving compared with living things at 
short exposure durations ( $20 \mathrm{msec}$ in their study) is due to their greater real-world structural variation than living things: Elephants have one basic real-world structural representation whereas telephones have many.

We present an alternative account of these category differences. At unlimited exposure durations, processing can be more difficult for living things relative to nonliving things because living things share many local parts in common with other members of the same category. Local parts take longer to be processed than more global aspects of shape such as outline contour (e.g., Eriksen, O'Hara, \& Eriksen, 1982; Hughes, Nozawa, \& Kitterle, 1996; Kimchi, 1992; Lupker, 1979; Navon, 1977; Sanocki, 1993; Townsend, $\mathrm{Hu}, \&$ Kadlec, 1988; Watt, 1988). It follows that a processing difficulty for living things will be more evident in tasks where local features are fully processed, such as semantic categorization and naming with unlimited exposure durations (Lloyd-Jones \& Humphreys, 1997a, 1997b) than in tasks where this may not be the case, such as naming at short exposure durations (e.g., Humphreys et al., 1995) and object decision (Lloyd-Jones \& Humphreys, 1997b). In contrast, with short exposure durations, a processing difficulty for nonliving things emerges because performance is based primarily on global aspects of shape and, in particular, the low diagnosticity of outline contour for recognition of nonliving things relative to living things increases processing time. We propose that outline contour information is more useful for the recognition of living things: There is a statistical regularity whereby usefulness of outline contour information for recognition correlates with object class. Evidence for outline contour mediating object recognition comes from experiments examining recognition across changes in viewpoint.

\section{The Importance of Outline Shape in Identifying Objects Across Changes in Viewpoint}

There is a continuing debate as to the kinds of features that mediate object recognition (e.g., Biederman \& Bar, 1999; Hayward \& Tarr, 2000). Biederman (1987; Hummel \& Biederman, 1992) proposes that the primary features used to identify objects are volumetric $3-\mathrm{D}$ primitives ("geons") that are fit to the visible parts of an object. However, although Biederman's theory has been very influential, the primary evidence showing viewpoint invariance in object recognition, as predicted by the theory, has been shown to have limited generalizability to other recognition tasks and stimulus sets (e.g., Hayward \& Tarr, 2000; Tarr \& Bülthoff, 1995; Tarr, Bülthoff, Zabinski, \& Blanz, 1997). Furthermore, Hayward (1998; Hayward \& Tarr, 1997) has shown that outline shape (bounding contour) is an important mediator of object recognition across changes in viewpoint. In sequential object matching and visual priming of objects in different orientations, performance with silhouettes was very similar (though not identical) to performance with shaded objects. This similarity in performance on the different stimuli suggests that visual features in the outline shape are important for object recog- nition. If silhouette recognition were markedly more difficult than recognition of fully shaded images ("shaded objects") in these paradigms, then we would have to conclude that nonsilhouette information, such as surface curvature (including geons) and texture information, is more important for object recognition. In fact, Hayward, Tarr, and Corderoy (1999) have found contributions of both outline contourand non-outline contourinformation to objectrecognition across depth rotation.

\section{The Present Experiment}

The following prediction arises from our account of category differences in object processing: When performance is mediated primarily by outline contour information, a processing difficulty for nonliving relative to living things will emerge. We examine this prediction using an object decision task (where participants decide whether the stimulus represents an object that could be encountered in real life) and a naming task, with both shaded objects and silhouettes.

For object decision, we propose that category differences, with longer responses to nonliving things, will emerge for both shaded objects and silhouettes, because outline contour information contributes to object decision performance. However, whereas non-outline contour information may also contribute to shaded object decision performance, for silhouette object decision, performance must be based on outline contour information alone. We therefore predict that category differences that arise due to differences in outline contour between the categories will be exaggerated in silhouette object decision relative to shaded object decision.

Evidence that object decision tasks need not require access to semantic or name information comes from the neuropsychologicalliterature, where patients have been shown to retain good performance on difficult object decision tests despite having marked impairments in their ability to name and retrieve associative information about objects (e.g., Chertkow, Bub, \& Caplan, 1992; Riddoch \& Humphreys, 1987; Sheridan \& Humphreys, 1993). Warrington and James (1988) have argued also that silhouette object decision (using foreshortened views of silhouetted objects and nonsense shapes having no identifiable object features) provides a measure of presemantic perceptual processing. Finally, converging evidence comes from Lupker (1988), who failed to find any semantic priming of object decision with a sequential presentation paradigm (as used here). On the basis of the foregoing arguments, we suggest that both shaded and silhouette object decision require the retrieval of stored structural object representations. Further processing, although possible, is not required (we shall return to this point in the Discussion).

For naming, we make the same predictions as for object decision. However, naming likely involves additional processes to object decision, which should be reflected in longer overall RTs (Lloyd-Jones \& Humphreys, 1997b). These additional processes may include retrieving seman- 
tic and name information, additional rate-limiting processes that may "wash out" effects of variables that have their locus at an earlier perceptual stage of processing.

As a subsidiary issue, we examine the effects of prior naming of shaded objects and silhouettes on object decision performance. This will allow us to determine (1) whether repetition priming (the facilitation in performance as a function of stimulus re-presentation) can reduce the processing disadvantage for nonliving things and (2) whether more general carryover effects (evidenced on both unprimed and primed target stimuli) can reduce the processing disadvantage for nonliving things.

A repetition priming paradigm was used. Many previous experiments examining visual object processing have used a basic study-test procedure (e.g., Biederman \& Cooper, 1991; Biederman \& Gerhardstein, 1993; LloydJones \& Humphreys, 1997a, 1997b). This experiment featured naming as the study phase task and object decision as the test phase task. The test phase followed immediately after the study phase. There are five important aspects to this paradigm: (1) It enabled us to examine category (living vs. nonliving) and stimulus (shaded vs. silhouette) differences in two tasks (naming and object decision); (2) it enabled us to examine effects of prior object processing (in naming) on retrieving stored structural representations of objects (in object decision); (3) repetition priming was examined across different tasks (objects were named on their initial presentation, whereas object decisions were made on all repeat presentations), thereby eliminating any task-specific components of the repetition effect (Monsell, 1985); (4) the initial block of items was always objects, so nonobject repetition effects were not observed and could not contribute to priming of shaded objects (e.g., if nonobjects shared features with objects); and (5) other target objects rather than primes acted as controls in a norepeat baseline condition against which priming was measured.

\section{METHOD}

\section{Participants}

There were 64 participants ( 32 male and 32 female). All were University of Kent undergraduate students, participating as partial fulfillment of a course requirement or for payment. All were native English speakers, with normal or corrected-to-normal vision, and all were naive as to the purpose of the experiment.

\section{Materials and Apparatus}

There were 64 line drawings of objects, 32 living and 32 nonliving (full lists are given in Appendix A). Drawings of objects were selected from the standardized set of Snodgrass and Vanderwart (1980). Living and nonliving objects were 64 of the 68 of those originally selected by Funnell and Sheridan (1992), who carefully matched living and nonliving objects for object complexity, familiarity, and name frequency (see also Stewart et al., 1992). In line with Tippett, Glosser, and Farah (1996; and more recently Laws \& Neve, 1999), we dropped "tree," which was classified as nonliving by Funnell and Sheridan, as well as "nose," "thumb," and "sun" (silhouettes of which independent judges decided were not nameable under untimed conditions). Examples of the Snodgrass and Vanderwart drawings, and their silhouettes, are given in Figure 1.
The three main visual variables that would be expected to influence object decision performance were object complexity, image agreement (which is assumed to reflect ease of matching a stored representation; Barry, Morrison, \& Ellis, 1997), and contour overlap (Humphreys et al., 1988). Statistical analyses confirmed that living and nonliving objects did not differ in terms of object complexity $[t(62)=1.28, p=.20]$, image agreement $[t(62)=0.58, p=.56]$, familiarity $[t(62)=.04, p=.96]$, or name frequency $[t(62)=.36$, $p=.717]$. However, the stimulus sets did differ in terms of contour overlap $[t(49)=3.21, p<.005]$. Ratings were not available for four living and nine nonliving items.

There were also 64 line drawings of non-objects taken from Kroll and Potter (1984). These non-objects have all the Gestalt properties of pictures of objects and so participants are not able to respond on the basis of gross figural differences between objects and non-objects.

Stimuli were presented on a PowerMac 8200/120 computer using SuperLab software (Version 1.5.7). For naming, latencies in vocalizing (measured in milliseconds) were obtained by interfacing the computer with a crystal clock and voice-activated relay. For object decision, keypresses activated computer-controlled collection of RTs. The timing cycle began with the onset of the target and terminated with the participant's response.

\section{Design}

A basic study-test procedure was used with naming as the study phase task and object decision as the test phase task. The variables of category (living vs. nonliving), priming (unprimed vs. primed), prime type (shaded vs. silhouette), and task type (shaded vs. silhouette object decision) were examined in four between-groups conditions manipulating the prime and target stimulus: (1) naming shaded/ object decision shaded, (2) naming silhouette/object decision shaded, (3) naming shaded/object decision silhouette, and (4) naming silhouette/object decision silhouette. Category and priming were within-subjects factors.
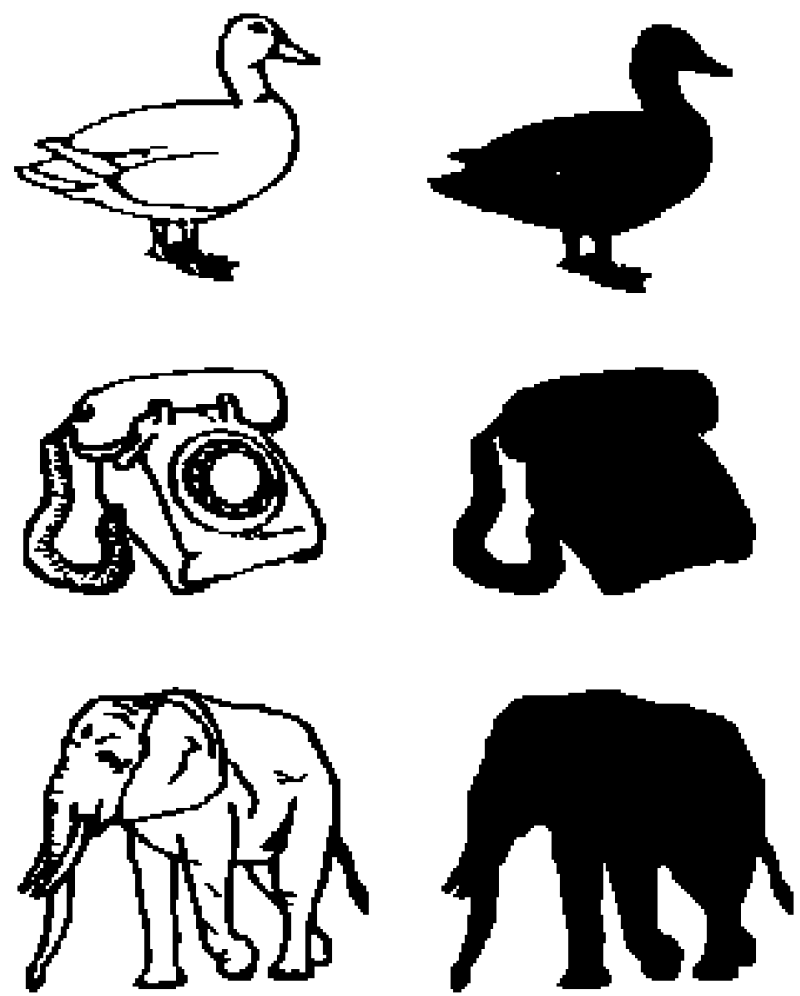

Figure 1. Examples of shaded objects and silhouettes. 
Table 1

Mean Response Times (RTs, in Milliseconds), Standard Errors (SEs), and Percentage Errors (PEs) for each of the Study Phase (Naming) Conditions for Shaded Objects $(\mathbf{R})$ and Silhouettes $(\mathrm{S})$ as Primes

\begin{tabular}{|c|c|c|c|c|c|c|c|c|c|c|c|c|}
\hline \multirow[b]{2}{*}{ Condition } & \multicolumn{3}{|c|}{$\mathrm{R}-(\mathrm{R})$} & \multicolumn{3}{|c|}{$S-(\mathrm{R})$} & \multicolumn{3}{|c|}{$\mathrm{R}-(\mathrm{S})$} & \multicolumn{3}{|c|}{$S-(S)$} \\
\hline & RT & $S E$ & PE & RT & $S E$ & $\mathrm{PE}$ & RT & $S E$ & $\mathrm{PE}$ & RT & $S E$ & PE \\
\hline Living & 810 & 28 & 1.9 & 930 & 36 & 12.8 & 910 & 31 & 8.4 & 887 & 30 & 8.8 \\
\hline Nonliving & 880 & 23 & 8.5 & 977 & 45 & 19.1 & 962 & 27 & 11.5 & 938 & 31 & 17.5 \\
\hline
\end{tabular}

Note-In the table, the letters in parentheses refer to the condition in which participants served in the subsequent object decision task.

To operationalize the experimental design, there were 32 living and 32 nonliving items. Each list of 32 items from each category was divided into two further equal lists (A and B) of 16 items, pairwise matched as far as possible in terms of means and ranges (for complexity, image agreement, familiarity, and name frequency). For each condition, half the 64 participants received List A of each category as the prime block (randomly ordered) with Lists A and B as the target block (randomly ordered). The other half received List B as the prime block and Lists A and B as the target block. Thus for each group of participants, half the targets were primed and half were unprimed. The prime block lasted approximately $4 \mathrm{~min}$ and the target block approximately $16 \mathrm{~min}$. The second block immediately followed the first. There were 32 naming trials in the prime block and 128 object decision trials in the target block ( 64 object trials and 64 non-object trials). For naming and object decision, there were 10 practice trials for each task immediately prior to the appropriate phase using items not otherwise encountered during the experiment (and equally from living and nonliving categories). Stimuli were presented until participants responded with a naming response (in the prime block) or keypress (in the target block). The participant responded in the target block by pressing either the " $\mathrm{z}$ " or the " $\mathrm{m}$ " on the keyboard, identifying the stimulus as either an "object" or a "non-object." The mapping of key onto response was counterbalanced and controlled for hand dominance.

\section{Results}

The mean correct RTs and errors for study and test phases were collated. The mean correct RTs, standard errors, and percentage errors for each condition in the study phase are given in Table 1, and for each condition in the test phase in Table 2. A trial was scored as an error if (1) participants gave an incorrect response, (2) the response latency was outside the criterion used for data trimming, or (3) a machine error occurred. In the error analysis, only trials falling into the first two categories were used. All data were trimmed using the nonrecursive moving criterion cutoff procedure of Van Selst and Jolicoeur (1994) to take sample size into account. Numbers 1 and 2 attached to the $F$ statistic refer to the by-participants and by-items analyses, respectively. If, due to errors and the deletion of outliers, a nil mean value was created for a particular item in the item analysis, in line with previous research practice this was subsequently replaced by the mean for that condition, to combat a loss of data (Tabachnick \& Fidell, 1996, p. 65). This resulted in dropping two items from the item analysis for naming (eye and accordion). Analyses dropping these items altogether produced the same results as reported below. We did not transform percentage error scores (e.g., with a log or arcsine transform); however, if we do so, the results remain unaltered.
We conducted three sets of analyses. First, we present analysis of the naming study phase data. Second, we present analysis of the object decision test phase (unprimed) baseline data. Third, we present the analyses examining the effect of prior naming on object decision. Note here that analyses of baseline object decision performance enable us to ensure that any effects of prior presentation on subsequent object decisions due to priming are not simply the result of a shift in baseline from one condition to another. In measuring priming, responses to target trials on which an error has been made to the stimulus on the corresponding study phase trial were not excluded (see Lloyd-Jones \& Humphreys, 1997b; Wheeldon \& Monsell, 1992). There was no evidence of a speed-accuracy tradeoff that would qualify the main interpretations of the RT data. We therefore give full statistical analyses of error scores in Appen$\operatorname{dix} B$.

\section{Study Phase: Naming}

Mean RTs and error scores for category (living vs. nonliving), prime type (shaded objects vs. silhouettes), and task type (whether participants went on to complete a shaded vs. silhouette object decision) were analyzed in a three-factor mixed analysis of variance (ANOVA). For all analyses the alpha level was set at .05. Planned comparisons used the cells means tests procedure advocated by Toothaker (1993, pp. 74-78).

There is evidence of longer naming times for nonliving compared with living things (although it is not robust across items). There is also evidence of longer RTs to silhouettes compared with shaded objects. However, this was evident for only half of the participants who went on to complete a shaded object decision task. ${ }^{2}$ The main results were (1) a main effect of category, by participants only, with shorter responses to living compared with nonliving things $\left[F_{1}(1,60)=18.80, M S_{\mathrm{e}}=5,152, F_{2}(1,62)=\right.$ $2.14, M S_{\mathrm{e}}=123,985$, n.s.], and (2) a prime type $\times$ task type interaction $\left[F_{1}(1,60)=4.88, M S_{\mathrm{e}}=28,604, F_{2}(1,62)\right.$ $\left.=19.88, M S_{\mathrm{e}}=60,471\right]$. Planned comparisons of the prime type $\times$ task type interaction revealed shorter RTs to shaded object primes compared with silhouette primes for the group that went on to complete the shaded object decision task, but not for the group that went on to complete the silhouette object decision task.

\section{Table 2}

Mean Response Times (RTs, in Milliseconds), Standard Errors $(S E s)$, and Percentage Errors (PEs) for each of the Test Phase (Object Decision) Conditions for Shaded Objects $(R)$ and Silhouettes $(S)$ as Targets

\begin{tabular}{|c|c|c|c|c|c|c|c|c|c|c|c|c|}
\hline \multirow[b]{2}{*}{ Condition } & \multicolumn{3}{|c|}{ (R)-R } & \multicolumn{3}{|c|}{ (S)-R } & \multicolumn{3}{|c|}{ (R)-S } & \multicolumn{3}{|c|}{$(\mathrm{S})-\mathrm{S}$} \\
\hline & RT & $S E$ & PE & RT & $S E$ & $\mathrm{PE}$ & RT & $S E$ & $\mathrm{PE}$ & RT & $S E$ & $\mathrm{PE}$ \\
\hline \multicolumn{13}{|l|}{ Living } \\
\hline Unprimed & 608 & 25 & 1.1 & 570 & 11 & 12.1 & 728 & 28 & 1.5 & 686 & 32 & 10.5 \\
\hline Primed & 557 & 16 & 1.5 & 556 & 9 & 7.4 & 651 & 20 & 0.4 & 649 & 38 & 7.4 \\
\hline \multicolumn{13}{|l|}{ Nonliving } \\
\hline Unprimed & 633 & 24 & 7.4 & 625 & 2 & 18.3 & 828 & 29 & 9.3 & 766 & 32 & 16.4 \\
\hline Primed & 596 & 17 & 3.9 & 606 & 19 & 10.5 & 740 & 27 & 4.6 & 675 & 33 & 13.6 \\
\hline
\end{tabular}

Note-In the table, the letters in parentheses refer to the condition in which participants served in the subsequent object decision task. 
There was also a main effect of prime type, by items only $\left[F_{1}(1,60)=2.02, M S_{\mathrm{e}}=28,604\right.$, n.s., $F_{2}(1,62)=45.17$, $\left.M S_{\mathrm{e}}=67,887\right]$, and a main effect of task type, by items only $\left[F_{1}(1,60)=0.71, M S_{\mathrm{e}}=28,604\right.$, n.s., $F_{2}(1,62)=$ $\left.14.89, M S_{\mathrm{e}}=67,277\right]$. There was no evidence of a category $\times$ prime type interaction $\left(F_{\mathrm{S}}<1\right)$.

\section{Test Phase: Object Decision Baselines}

Mean baseline RTs and error scores for category (living vs. nonliving), prime type (shaded vs. silhouette), and task type (shaded vs. silhouette object decision) were analyzed in a three-factor mixed ANOVA.

For object decision, there was evidence of a category difference in both shaded object decision and silhouette object decision, with longer RTs for nonliving compared with living things. Importantly, the category difference was greater for silhouette object decision. The main results were (1) a main effect of category with shorter RTs to living compared with nonliving things $\left[F_{1}(1,60)=\right.$ $\left.28.95, M S_{\mathrm{e}}=6,139, F_{2}(1,62)=12.43, M S_{\mathrm{e}}=76,650\right]$, and $(2)$ a category $\times$ task type interaction $\left[F_{1}(1,60)=\right.$ $6.19, M S_{\mathrm{e}}=6,139, F_{2}(1,62)=3.86, p=.05, M S_{\mathrm{e}}=$ $50,723]$. Planned comparisons of the category $\times$ task type interaction revealed shorter RTs to living compared with nonliving things for both shaded object decision and silhouette object decision. The interaction arose because the difference between living and nonliving things was greater for silhouette object decision (a 90-msec difference for silhouette object decision vs. a 40-msec difference for shaded object decision). We can analyze this interaction using a within-categories baseline and produce the same interpretation. Planned comparisons revealed shorter RTs to shaded object decision compared with silhouette object decision for both nonliving and living things. The interaction then arose because the difference between shaded object decision and silhouette object decision was greater for nonliving things $(168 \mathrm{msec})$ than for living things (118 msec).

There was also a main effect of task type, with shorter RTs to shaded object decision compared with silhouette object decision $\left[F_{1}(1,60)=37.80, M S_{\mathrm{e}}=19,687, F_{2}(1,62)=\right.$ 33.70, $\left.M S_{\mathrm{e}}=50,723\right]$.

\section{Test Phase: Object Decision (Priming)}

Mean RTs and error scores for category (living vs. nonliving), priming (primed vs. unprimed), prime type (shaded vs. silhouette), and task type (shaded vs. silhouette object decision) were analyzed in a four-factor mixed ANOVA.

First, consistent with previous results, there was evidence of a category difference, with longer RTs for nonliving compared with living things, in both shaded object decision and silhouette object decision. Importantly, this category difference was greater in silhouette object decision. The main results were (1) a main effect of category, with shorter RTs to living compared with nonliving things $\left[F_{1}(1,60)=97.16, M S_{\mathrm{e}}=2,215, F_{2}(1,62)=5.30, M S_{\mathrm{e}}=\right.$ $81,915]$, and (2) a category $\times$ task type interaction, by participants only $\left[F_{1}(1,60)=6.93, M S_{\mathrm{e}}=2,215, F_{2}(1,62)=\right.$
$1.65, M S_{\mathrm{e}}=35,628$, n.s.]. Planned comparisons of the category $\times$ task type interaction by participants revealed longer RTs to nonliving compared with living things for both shaded object decision and silhouette object decision. The interaction arose because the difference between nonliving and living things was greater for silhouette object decision ( $73 \mathrm{msec}$ ) than for shaded object decision (42 msec). If we analyze this interaction using a within-categories baseline, we produce the same interpretation (and the mean difference is the same). Planned comparisons revealed shorter RTs to shaded object decision compared with silhouette object decision for both nonliving and living things. The interaction arose because the difference between shaded object decision and silhouette object decision was greater for nonliving things $(137 \mathrm{msec})$ than for living things (106 msec).

Second, priming was equivalent for living and nonliving things: There was a main effect of priming, not qualified by category, with shorter RTs for primed compared with unprimed stimuli $\left[F_{1}(1,60)=58.12, M S_{\mathrm{e}}=2,955\right.$, $\left.F_{2}(1,62)=30.76, M S_{\mathrm{e}}=10,130\right]$.

Third, longer silhouette object decisions to nonliving compared with living things were reduced by silhouette primes relative to shaded object primes. This was the case for both unprimed and primed stimuli. There was a category $\times$ task type $\times$ prime type interaction $\left[F_{1}(1,60)=\right.$ $\left.6.86, M S_{\mathrm{e}}=2,215, F_{2}(1,62)=4.51, M S_{\mathrm{e}}=16,872\right]$. This interaction was analyzed further in two separate ANOVAs for each task type (shaded vs. silhouette object decision). When the task was shaded object decision, there was a main effect of category, with shorter RTs to living compared with nonliving things $\left[F_{1}(1,30)=26.72, M S_{\mathrm{e}}=\right.$ $\left.2,163, F_{2}(1,62)=7.17, M S_{\mathrm{e}}=56,692\right]$. There was also a main effect of priming, with shorter RTs to primed compared with unprimed stimuli $\left[F_{1}(1,30)=12.75, M S_{\mathrm{e}}=\right.$ $\left.2,335, F_{2}(1,62)=11.08, M S_{\mathrm{e}}=9,016\right]$. When the task was silhouette object decision, there was a main effect of category, with shorter RTs to living compared with nonliving things, by participants only $\left[F_{1}(1,30)=76.20, M S_{\text {e }}\right.$ $=2,268, F_{2}(1,62)=1.42, M S_{\mathrm{e}}=60,851$, n.s.]. There was a main effect of priming, with shorter RTs to primed compared with unprimed stimuli $\left[F_{1}(1,30)=47.83, M S_{\mathrm{e}}=\right.$ $\left.3,575, F_{2}(1,62)=15.06, M S_{\mathrm{e}}=14,874\right]$. There was also a category $\times$ prime type interaction $\left[F_{1}(1,30)=6.05\right.$, $\left.M S_{\mathrm{e}}=2,268, F_{2}(1,62)=5.49, M S_{\mathrm{e}}=26,714\right]$. Planned comparisons of the category $\times$ prime type interaction revealed shorter RTs to living compared with nonliving things for both shaded object and silhouette primes. The interaction arose because the difference between living and nonliving things was reduced following silhouette primes (53 msec) compared with shaded object primes (95 msec).

Fourth, silhouette object decision takes longer than shaded object decision and there is also more priming for silhouette object decision. There was (1) a main effect of task type, with shorter RTs for shaded object decision compared with silhouette object decision $\left[F_{1}(1,60)=\right.$ $\left.32.53, M S_{\mathrm{e}}=29,046, F_{2}(1,62)=67.15, M S_{\mathrm{e}}=35,628\right]$, 
and (2) a task type $\times$ priming interaction $\left[F_{1}(1,60)=\right.$ $\left.9.81, M S_{\mathrm{e}}=2,955, F_{2}(1,62)=11.65, M S_{\mathrm{e}}=16,872\right]$. Planned comparisons of the task type $\times$ priming interaction revealed priming for both shaded object decision and silhouette object decision. The interaction arose because there was more priming for silhouette object decision $(73 \mathrm{msec})$ than for shaded object decision $(30 \mathrm{msec})$.

Finally, there were shorter RTs for the group that in the study phase received silhouette compared with shaded $o b$ ject primes (by items only): There was a main effect of prime type $\left[F_{1}(1,60)=1.53, M S_{\mathrm{e}}=29,046\right.$, n.s., $F_{2}(1,62)=5.93$, $\left.M S_{\mathrm{e}}=14,932\right]$.

\section{Summary of Main Results}

The main results are as follows: First, there were longer RTs and less accuracy for silhouettes compared with shaded objects, and these stimulus differences were more pronounced for object decision than for naming: (1) For naming, the differences were evident only for a subset of participants in the prime phase naming analysis, and (2) stimulus differences were larger for object decision (143 msec) compared with naming (42 msec) for naming (see Appendix C for a direct statistical comparison). Second, there were longer RTs and less accuracy for nonliving compared with living things, and these category differences were more pronounced for object decision than naming: (1) Although the category RT difference was similar for the two tasks ( $55 \mathrm{msec}$ for naming, $65 \mathrm{msec}$ for object decision baselines), the category differences for naming were true only for a subset of items, and (2) whereas for naming there were longer RTs to nonliving compared with living things, which were equivalent for shaded objects and silhouettes (61 vs. $49 \mathrm{msec}$, respectively), for object decision there were longer RTs to nonliving compared with living things for silhouettes and shaded objects, and the difference was greater for silhouettes ( $90 \mathrm{vs.} 40 \mathrm{msec}$, respectively). These task differences were confirmed statistically in an analysis directly comparing naming and object decision baselines (full analyses are given in Appen$\operatorname{dix} C)$.

\section{DISCUSSION}

\section{Object Decision and Naming}

For object decision, but not for naming, changing a picture from a shaded object to a silhouette increased processing difficulty for both categories of object, and it did so more for nonliving than for living things. This suggests that outline shape is more important in the recognition of living things, when a participant is deciding whether the stimulus is an object. Although RTs to nonliving things were longer than to living things for both shaded and silhouette object decision, when task performance was dependent solely on outline shape (as in silhouette object decision), processing efficiency for nonliving things was reduced to a greater extent than for living things.

These results suggest that object decision was more sensitive to the category and stimulus differences observed here than was naming. They are also consistent with cat- egory and stimulus differences arising at an early level of processing. Naming likely involves additional processes to object decision, as evidenced by longer naming than object decision RTs. These additional processes may include retrieving semantic and name information, additional ratelimiting processes that may "wash out" effects of variables that have their locus at an earlier perceptual stage of processing.

Finally, let us examine one other aspect of object decision performance. Silhouette object decision takes longer than shaded object decision. This is consistent with both outline contour and non-outline contour information (including internal features and surface properties) contributing to normal object recognition. When both kinds of information are available, performance is more efficient than with outline contour information alone (Hayward et al., 1999). Nevertheless, outline contour information alone can successfully mediate object recognition and naming. The longer initial baselines for silhouette object decision likely reflect increased visual processing relative to that required for shaded object decision, and these longer initial baselines allow more room for improvement and hence more priming. However, these increased RTs and greater priming may reflect the fact that silhouette object decision involves an additional process to those involved in shaded object decision-either the retrieval of semantic information (to differentiate objects from nonobjects when less information is available in the display) or some kind of "double checking" procedure (see Corballis, 1988).

\section{Effects of Prior Naming on Object Decision}

The main results are as follows: (1) Priming was equivalent for nonliving and living objects for both shaded object decision and silhouette object decision (despite differences in baseline RTs) and did not reduce the object decision disadvantage for nonliving things relative to living things, and (2) the nature of the prime (a shaded object or silhouette) did not influence subsequent shaded object decision performance. However, importantly, naming silhouette primes, relative to shaded primes, reduced the disadvantage for nonliving things in silhouette object decision. This was the case for objects not encountered before as well as for those seen in the prime phase. Participants therefore learned some general ability to perceive outline contour, which mitigates the initial disadvantage for nonliving things in silhouette object decision.

These category differences in object decision and naming baselines and effects of the prime on subsequent performance are not due to preexisting differences between the stimulus lists in object complexity, familiarity, or name frequency. Similarly, they cannot be due to differences in image agreement (since lists were matched on this variable as well). Barry et al. (1997) have argued that this variable reflects the ease of matching input to a stored structural representation. If this is the case, and if the Laws and Neve (1999) account is correct (i.e., that poorer accuracy for nonliving things is due to their greater real-world structural variation), we should not have observed cate- 
gory differences in the present experiment. The stimulus lists did differ in contour overlap, however, with greater contour overlap for living things. Objects with greater contour overlap and hence more structurally similar neighbors may be argued to be easier to classify as "objects" compared with non-objects. Nevertheless, if we statistically partial out contour overlap in an analysis of covariance on object decision baseline scores, the relation between category and stimulus remains [i.e., there is a category $\times$ task type interaction $F(1,56)=4.38, M S_{\mathrm{e}}=$ 54,624].

One final concern is the inclusion of body parts as living things and musical instruments as nonliving things in the present study. In the neuropsychological literature, musical instrument naming has been found to be impaired with living things, whereas body part naming has been found to be impaired with nonliving things (see, e.g., Saffran \& Schwartz, 1994, for a review). To be able to relate the present results directly to the neuropsychological literature, we need to show that the general pattern of results remains when those items are excluded from the present analyses. This indeed turns out to be the case: Category and stimulus differences remain. ${ }^{3}$

\section{Conclusion}

We suggest two possibilities as to why (1) the outline shape of nonliving things relative to living things makes deciding whether they are objects particularly difficult, and (2) prior experience of naming silhouettes rather than shaded objects reduces the difficulty for nonliving objects in silhouette object decision (for both those encountered before and those seen for the first time). First, there may be less useful information in the outline contour for nonliving compared with living things. They may possess fewer salient object features that therefore require more precise visual discriminations (see Hoffman \& Singh, 1997, for a discussion of feature salience). Prior silhouette naming, which requires precise visual discriminations to match the object to a unique label, increases expertise, which subsequently benefits object decision. Prior studies are consistent with this idea. For instance, Doane, Alderton, Sohn, and Pellegrino (1996) found that exposure to highly visually similar sets of stimuli requiring difficult visual discriminations led eventually to shorter RTs, more accurate discriminations, and superior transfer performance (relative to stimuli allowing easy visual discriminations) on novel stimuli. An alternative possibility is that there may be greater 2-D/3-D interpretational ambiguity for the outline shapes of nonliving relative to living things; the outline shapes of nonliving things may be closer to being "impossible objects" (see Richards, Koenderink, \& Hoffman, 1987; Walker \& Walker, 1988). Under this view, prior silhouette naming, which requires precise visual processing, increases expertise in the process of 2-D/3-D interpretation.

Lloyd-Jones and Humphreys (1997a, 1997b) have suggested that neurological impairments may exacerbate previously existing normal processing differences. We present a similar case here. When perceptual input is degraded through neurological impairment, resulting in reliance on global as opposed to local featural information, a categoryspecific deficit may emerge for nonliving things. Such a deficit is not an indication of a difference in the stored representation of specific categories of items, but rather results from the correlation between the importance of outline contour information for recognition and object class. Thus, category specificity in neuropsychological cases need not indicate a difference in the nature of the stored representations of specific categories of items. Rather, processing differences between categories, which exist in normality, can contribute to observed behavior.

\section{REFERENCES}

Barry, C., Morrison, C. M., \& Ellis, A. W. (1997). Naming the Snodgrass and Vanderwart pictures: The effects of name age-of-acquisition, frequency and agreement. Quarterly Journal of Experimental Psychology, 50A, 560-585.

Biederman, I. (1987). Recognition by components: A theory of human image understanding. Psychological Review, 94, 115-145.

Biederman, I., \& BAR, M. (1999). One-shot viewpoint invariance in matching novel objects. Vision Research, 17, 2885-2899.

Biederman, I., \& CoOper, E. E. (1991). Evidence for complete translational and reflectional invariance in visual object priming. Perception, 20, 585-593.

Biederman, I., \& Gerhardstein, P. C. (1993). Recognizing depth-rotated objects: Evidence for 3D viewpoint invariance. Journal of Experimental Psychology: Human Perception \& Performance, 19, 1162 1182.

Chertkow, H., Bub, D., \& Caplan, D. (1992). Constraining theories of semantic memory processing: Evidence from dementia. Cognitive Neuropsychology, 9, 327-365.

Corballis, M.C. (1988). Recognition of disoriented shapes. Psychological Review, 95, 115-123.

Donne, S. M., Alderton, D. L., Sohn, Y. W., \& Pellegrino, J. W. (1996). Acquisition and transfer of skilled performance: Are visual discrimination skills stimulus specific? Journal of Experimental Psychology: Human Perception \& Performance, 22, 1218-1248.

Eriksen, C. W., O'Hara, W. P., \& Eriksen, B. A. (1982). Response competition effects in same-different judgments. Perception \& Psychophysics, 32, 261-270.

Farah, M. J., McMullen, P. A., \& Meyer, M. M. (1991). Can recognition of living things be selectively impaired? Neuropsychologia, 29, 185-193.

Farah, M. J., \& Wallace, M. A. (1992). Semantically bounded anomia: Implications for the neural implementation of naming. $\mathrm{Neu}$ ropsychologia, 30, 609-621.

Funnell, E., \& Hodges, J. R. (1996). Deficits of semantic memory end executive control: Evidence for differing effects upon naming in dementia. Aphasiology, 10, 687-709.

Funnell, E., \& Sheridan, J. (1992). Categories of knowledge? Unfamiliar aspects of living and nonliving things. Cognitive Neuropsychology, 9, 135-153.

Gaffan, D., \& Heywood, C. A. (1993). A spurious category-specific visual agnosia for living things in normal human and nonhuman primates. Journal of Cognitive Neuroscience, 5, 118-128.

GainotTi, G., \& Silveri, M. C. (1996). Cognitive and anatomical locus of lesion in a patient with a category-specific semantic impairment for living beings. Cognitive Neuropsychology, 13, 357-390.

Hart, J., Berndt, R. S., \& Caramazza, A. C. (1985). Category-specific naming deficit following cerebral infarction. Nature, 316, 439-440.

HART, J., \& Gordon, B. (1992). Neural subsystems for object knowledge. Nature, 359, 60-64.

HAYWARD, W. G. (1998). Effects of outline shape in object recognition. Journal of Experimental Psychology: Human Perception \& Performance, 24, 427-440.

HAYWARD, W. G., \& TARR, M. J. (1997). Testing conditions for viewpoint invariance in object recognition. Journal of Experimental Psychology: Human Perception \& Performance, 23, 1511-1521. 
Hayward, W. G., \& TARr, M. J. (2000) Differing views on views: Comments on Biederman and Bar (1999). Vision Research, 40, 3895-3899.

Hayward, W. G., Tarr, M. J., \& Corderoy, A. K. (1999). Recognizing silhouettes and shaded images across depth rotation. Perception, 28, 1197-1215.

Hillis, A. E., \& Caramazza, A. (1991). Category-specific naming and comprehension impairment: A double dissociation. Brain, 114, 20812094.

Hoffman, D. D., \& Singh, M. (1997). Salience of visual parts. Cognition, 63, 29-78.

Hughes, H. C., Nozawa, G., \& Kitterle, F. (1996). Global precedence, spatial frequency channels, and the statistics of natural images. Journal of Cognitive Neuroscience, 8, 197-230.

Hummel, J. E., \& Biederman, I. (1992). Dynamic binding in a neural network for shape recognition. Psychological Review, 99, 480-517.

Humphreys, G. W., Lloyd-Jones, T. J., \& Fias, W. (1995). Semantic interference effects on naming using a postcue procedure: Tapping the links between semantics and phonology with pictures and words. Journal of Experimental Psychology: Learning, Memory, \& Cognition, 21, 961-980.

Humphreys, G. W., Riddoch, M. J., \& Quinlan, P. T. (1988). Cascade processes in picture identification. Cognitive Neuropsychology, 5, 67 103.

KIмchI, R. (1992). Primacy of wholistic processing and global/local paradigm: A critical review. Psychological Bulletin, 112, 24-38.

Kroll, J. F., \& Potter, M. C. (1984). Recognizing words, pictures, and concepts: A comparison of lexical, object, and reality decisions. Journal of Verbal Learning, \& Verbal Behavior, 23, 39-66.

Lambon-Ral Ph, M. A., Howard, D., Nightingale, G., \& Ellis, A. W. (1998). Are living and nonliving category-specific deficits causally linked to impaired perceptual or associative knowledge? Evidence from a category-specific double dissociation. Neurocase, 4, 311-338

Laws, K. R., \& Neve, C. (1999). A "normal” category-specific advantage for naming living things. Neuropsychologia, 37, 1263-1269.

Lloyd-Jones, T. J., \& Humphreys, G. W. (1997a). Categorizing chairs and naming pears: Category differences in object processing as a function of task and priming. Memory \& Cognition, 25, 606-624.

Lloyd-Jones, T. J., \& Humphreys, G. W. (1997b). Perceptual differentiation as a source of category effects in object processing: Evidence from naming and object decision. Memory \& Cognition, 25, 18-35.

LUCKHURST, L., \& LLOYD-Jones, T. J. (2001). A selective deficit for living things after temporal lobectomy for relief of epileptic seizures. Brain \& Language, 79, 266-296.

LUPKER, S. J. (1979). The semantic nature of response competition in the picture-word interference task. Memory \& Cognition, 7, 485-495.

LUPKER, S. J. (1988). Picture naming: An investigation of the nature of categorical priming. Journal of Experimental Psychology: Learning, Memory, \& Cognition, 14, 444-455.

Monsell, S. (1985). Repetition and the lexicon. In A. W. Ellis (Ed.), Progress in the psychologyoflanguage(Vol. 2, pp. 147-195).London: Erlbaum.

Navon, D. (1977). Forest before trees: The precedence of global features in visual perception. Cognitive Psychology, 9, 353-383.

Richards, W. A,. Koenderink, J. J., \& Hoffman, D. D. (1987). Inferring three-dimensional shapes from two-dimensional silhouettes. Journal of the Optical Society of America A, 4, 1168-1175.

Riddoch, M. J., \& Humphreys, G. W. (1987). Visual object processing in optic aphasia: A case of semantic access agnosia. Cognitive Neuropsychology, 4, 131-185.

Sacchett, C., \& Humphreys, G. W. (1992). Calling a squirrel a squirrel, but a canoe a wigwam: A category-specific deficit for artefactual objects and body parts. Cognitive Neuropsychology, 9, 73-86.

Saffran, E. J., \& Schwartz, M. F. (1994). Of cabbages and things: Semantic memory from a neuropsychological point of view-A tutorial review. In C. Umiltà \& M. Moscovitch (Eds.), Attention and Performance, XV: Conscious and nonconscious information processing. (pp. 507-536). Cambridge, MA: MIT Press, Bradford Books.

SAmson, D., Pillon, A., \& De Wilde, V. (1998). Impaired knowledge and non-visual attributes in a patient with a semantic impairment for living entities: A case of true category-specific deficit. Neurocase, 4, 273-290.

SANOCKI, T. (1993). Time course of object identification: Evidence for a global-to-local contingency. Journal of Experimental Psychology: Human Perception \& Performance, 19, 878-898.

SARTORI, G., \& Job, R. (1988). The oyster with four legs: A neuropsychological study on the interaction of visual and semantic information. Cognitive Neuropsychology, 5, 105-132.

Sartori, G., Job, R. \& Coltheart, M. (1993). The organization of object knowledge: Evidence from neuropsychology. In D. E. Meyer \& S. Kornblum (Eds.), Attention and Performance XIV: Synergies in experimental psychology, artificial intelligence, and cognitive neuroscience. (pp. 451-465). Cambridge, MA: MIT Press.

SheridAN, J., \& Humphreys, G. W. (1993). A verbal-semantic categoryspecific recognition impairment. Cognitive Neuropsychology, 10, 143 184.

Silveri, M. C., \& GainotTi, G. (1988). Interaction between vision and language in category-specific semantic impairment. Cognitive $\mathrm{Neu}$ ropsychology, 5, 677-709.

Snodgrass, J. G., \& VANDERWARt, M. (1980). Standardized set of 260 pictures: Norms of name agreement, usage agreement, familiarity, and visual complexity. Journal of Experimental Psychology: Human Learning \& Memory, 6, 174-215.

Stewart, F., Parkin, A. J., \& Hunkin, N. M. (1992). Naming impairments following recovery from herpes simplex encephalitis: Categoryspecific? Quarterly Journal of Experimental Psychology, 44A, 261284.

TAвAChnick, B. G., \& Fidell, L. S. (1996). Using multivariate statistics (3rd Ed). New York: HarperCollins.

TARR, M. J., \& BÜLThOFF, H. H. (1995). Is human object recognition better described by geon structural descriptions or by multiple views? Comment on Biederman and Gerhardstein (1993). Journal of Experimental Psychology: Human Perception \& Performance, 21, 14941505.

Tarr, M. J., Bülthoff, H. H., Zabinski, M., \& Blanz, V. (1997). To what extent do unique parts influence recognition across changes in viewpoint? Psychological Science, 8, 282-289.

Tippett, L. J., Glosser, G., \& FARAH, M. J. (1996). A category-specific naming impairment after temporal lobectomy. Neuropsychologia, 34, 139-146.

TOOTHAKER, L. E. (1993). Multiple comparison procedures. (Quantitative Applications in the Social Science, No. 89). Newbury Park, CA: Sage.

Townsend, J. T., Hu, G. G., \& Kadlec, H. (1988). Feature sensitivity, bias, and interdependencies as a function of energy and payoffs. Perception \& Psychophysics, 43, 575-592.

VAn Selst, M., \& Jolicoeur,P. (1994). A solution to the effect of sample size on outlier elimination. Quarterly Journal of Experimental Psychology, 47A, 631-650.

WALKer, J. T., \& WALKer, M. J. (1988). The apparent size of threedimensional objects and their silhouettes: A solid-superiority effect. Perception, 17, 23-30.

WARRINGTON, E. K., \& JAMES, M. (1988). Visual apperceptive agnosia: A clinico-anatomical study of three cases. Cortex, 24, 13-32.

Warrington, E. K., \& McCARThy, R. (1983). Category-specific access dysphasia. Brain, 106, 859-878.

WARrington,E. K., \& MCCARTHY, R. (1987).Categories of knowledge: Further fractionation and an attempted integration. Brain, 110, 12731296.

Warrington, E. K., \& Shallice, T. (1984). Category-specific semantic impairment. Brain, 107, 829-853.

WATT, R. J. (1988). Visual processing: Computational, psychophysical and cognitive research. London: Erlbaum.

WheELdon, L. R. \& Monsell, S. (1992). The locus of repetition priming of spoken word production. Quarterly Journal of Experimental Psychology, 44A, 723-761.

\section{NOTES}

1. Gaffan and Heywood (1993) found longer RTs to living than to nonliving things with a speeded presentation paradigm. However, the categories used in their experiment were not matched for important variables that may produce category differences in performance (e.g., object complexity and familiarity). 
2. A potential problem is that in naming (1) a nonliving-living difference (although only true for a subset of items) and (2) a shaded objectsilhouette difference may have important consequences for subsequent object decision performance. However, this is not the case: (1) Priming was equivalent for nonliving and living things; (2) an overall difference between nonliving and living things in naming cannot explain longer responses to nonliving things in silhouette object decision, but not shaded object decision, which are reduced by a silhouette and not a shaded object prime; and (3) the shaded object-silhouette difference in naming was apparent for the group that went on to make object decisions to shaded objects. However, this group showed no effect of shaded versus silhouette primes in shaded object decision (i.e., there was no main effect of prime type or interaction with prime type).

The prime type $\times$ task type interaction in naming was significant at the $p=.03$ level, and may have arisen due to either 1 participant being particularly efficient in the shaded object/shaded object condition (1 participant in particular recorded responses of $<700 \mathrm{msec}$ ) or 1 participant being particularly inefficient in the silhouette/shaded object condition (1 participant in particular recorded responses of $>1,200 \mathrm{msec}$ ). If either of these participants is dropped from the analysis, the interaction disappears.

3 . There is some evidence of more priming for living things than nonliving things for shaded object decision, but equivalent priming for silhouette object decision. These results therefore do not alter our interpretation of the present findings. The main difference between the two sets of analyses is that when musical instruments and body parts are dropped, we lose the category $\times$ prime type $\times$ task type interaction, showing a reduction in the relative disadvantage for nonliving things in silhouette object decision, by a silhouette as compared with a shaded object prime. It may be the case that prior exposure to musical instruments in particular leads to better general learning of some ability to perceive outline contour, which particularly benefits nonliving things. However, we have no reason to believe this. Rather, we argue that losing the three-way interaction is simply a reflection of a less powerful analysis that includes only $84.4 \%$ of the original items. Full data are available on request.

\section{APPENDIX A}

Stimuli

\section{Living}

arm, banana, cherry, dog, duck, elephant, eye, hand, leaf, lobster, mouse, pear, pig, potato, squirrel, zebra, apple, carrot, cat, celery, donkey, ear, foot, frog, grapes, lips, monkey, onion, rhino, spider, tomato, turtle.

\section{Nonliving}

accordion, anchor, chair, cigar, couch, door, drum, flute, fork, glass, glove, hammer, needle, ruler, scissors, thimble, arrow, axe, balloon, bicycle, broom, button, cannon, harp, kite, knife, pliers, plug, shirt, telephone, umbrella, whistle.

\section{APPENDIX B}

Error Analyses

\section{Study Phase: Naming}

There was a main effect of category, with more errors to nonliving compared with living things $\left[F_{1}(1,60)=57.23, M S_{\mathrm{e}}=\right.$ $\left.85.90, F_{2}(1,62)=6.72, M S_{\mathrm{e}}=172\right]$. There was a main effect of prime type, with more errors to silhouettes than to shaded objects $\left[F_{1}(1,60)=46.25, M S_{\mathrm{e}}=134.90, F_{2}(1,62)=58.97, M S_{\mathrm{e}}=70.80\right]$. There was a category $\times$ prime type interaction, by items only $\left[F_{1}(1,60)=2.58, M S_{\mathrm{e}}=85.90\right.$, n.s., $F_{2}(1,62)=6.71, M S_{\mathrm{e}}=$ $70.80]$. There was also a main effect of task type, with more errors for the group that went on to complete the silhouette object decision task compared with the shaded object decision task, by items only $\left[F_{1}(1,60)=0.81, M S_{\mathrm{e}}=134\right.$, n.s., $F_{2}(1,62)=7.12, M S_{\mathrm{e}}=$ $70.80]$. Finally, there was a prime type $\times$ task type interaction $\left[F_{1}(1,60)=13.41, M S_{\mathrm{e}}=134.90, F_{2}(1,62)=18.41, M S_{\mathrm{e}}=31.21\right]$. The three-way prime type $\times$ task type $\times$ category interaction was marginally significant by participants only $\left[F_{1}(1,60)=3.41\right.$, $M S_{\mathrm{e}}=85.90, p=.06, F_{2}(1,62)=1.91, M S_{\mathrm{e}}=31.20$, n.s. $]$.

Planned comparisons of the category $\times$ prime type interaction, by items only, revealed more errors to silhouettes compared with shaded objects for nonliving things $(p<.01)$. Planned comparisons of the prime type $\times$ task type interaction revealed more errors to silhouettes compared with shaded objects for the group that went on to complete the shaded object decision task ( $p<.01$ by participants and by items). The trend toward a prime type $\times$ task type $\times$ category interaction by participants arose because the pattern of more errors to silhouettes compared with shaded objects for nonliving things was most pronounced for the group that went on to complete the shaded object decision task.

\section{Test Phase: Object Decision Baselines}

There was a main effect of category, marginally significant by items, with more errors for nonliving compared with living things $\left[F_{1}(1,60)=22.63, M S_{\mathrm{e}}=1.54, F_{2}(1,62)=3.20, M S_{\mathrm{e}}=\right.$ $5.63, p=.07]$. There was also a main effect of prime type, with more errors for silhouettes compared with shaded objects as primes $\left[F_{1}(1,60)=27.92, M S_{\mathrm{e}}=2.63, F_{2}(1,62)=15.74, M S_{\mathrm{e}}=\right.$ 2.19].

\section{Across Task Comparison: Naming Versus Object Decision Baselines}

There was a main effect of category, by participants only, with more errors to nonliving compared with living things $\left[F_{1}(1,30)=\right.$ $98.85, M S_{\mathrm{e}}=37.35, F_{2}(1,62)=2.13, M S_{\mathrm{e}}=4.76$, n.s.]. There was also a main effect of type, with more errors to silhouettes compared with shaded objects $\left[F_{1}(1,30)=73.14, M S_{\mathrm{e}}=68.35\right.$, $\left.F_{2}(1,62)=21.52, M S_{\mathrm{e}}=3.60\right]$.

\section{Test Phase: Object Decision Priming}

There was a main effect of category, with more errors to nonliving compared with living things, by participants only $\left[F_{1}(1,60)=\right.$ $\left.35.77, M S_{\mathrm{e}}=49.74, F_{2}(1,62)=2.81, M S_{\mathrm{e}}=7.65, p=.09\right]$. There was also a main effect of priming, with more errors to unprimed compared with primed stimuli $\left[F_{1}(1,60)=11.84, M S_{\mathrm{e}}=\right.$ $\left.63.13, F_{2}(1,62)=13.08, M S_{\mathrm{e}}=0.75\right]$. Finally, there was a main effect of prime type, with more errors to silhouettes compared with shaded objects as primes $\left[F_{1}(1,60)=59.49, M S_{\mathrm{e}}=74.11\right.$, $\left.F_{2}(1,62)=15.92, M S_{\mathrm{e}}=3.25\right]$. 
APPENDIX C

\section{Across Task Comparison: Naming Versus Object Decision Baselines}

To directly compare naming and object decision baseline performance, we compared baseline scores from the study and test phases of two conditions: (1) naming shaded-object decision shaded and (4) naming silhouette-object decision silhouette. This between-groups comparison represents a conservative test of differences between conditions.

Mean baseline RTs and error scores for category (living vs. nonliving), type (shaded vs. silhouette), and task (naming vs. object decision) were analyzed in a three-factor mixed ANOVA. The comparison was between baseline scores from the study and test phases of two conditions:(1) naming shaded-object decision shaded (unprimed), and (4) naming silhouette-object decision silhouette (unprimed).

A direeteomparison of naming and object decisioneonfirms the result of each separate task analysis: There is evidence for a category difference, with shorter RTs to living compared with nonliving things, and a trend toward this difference being most pronounced for object decisions to silhouettes. The main results are therefore (1) a main effect of category, with shorter RTs to living compared with nonliving things $\left[F_{1}(1,30)=28.24, M S_{\mathrm{e}}=\right.$ $\left.4,267, F_{2}(1,62)=8.91, M S_{\mathrm{e}}=72,920\right]$, and (2) a three-way category $\times$ type $\times$ task interaction, marginally significant by participants only $\left[F_{1}(1,30)=3.54, M S_{\mathrm{e}}=4,869, p=.06\right.$,
$F_{2}(1,62)=0.81, M S_{\mathrm{e}}=17,908$, n.s.]. The trend toward a threeway category $\times$ type $\times$ task interaction arose because for object decision there were shorter RTs to living compared with nonliving things for silhouettes but not shaded objects (i.e., there was a category $\times$ type interaction for object decision but not naming).

There were also longer RTs for naming compared with object decision, and longer RTs for silhouettes compared with shaded objects. The difference between silhouettes and shaded objects was more pronounced for object decision than naming. Thus, there was a main effect of task, with shorter RTs for object decision compared with naming $\left[F_{1}(1,30)=43.85, M S_{\mathrm{e}}=29,351\right.$, $\left.F_{2}(1,62)=71.35, M S_{\mathrm{e}}=36,516\right]$. There was also a main effect of type, with shorter RTs to shaded objects empared with sithouettes $\left[F_{1}(1,30)=14.14, M S_{\mathrm{e}}=18,805, F_{2}(1,62)=31.67\right.$, $\left.M S_{\mathrm{e}}=39,900\right]$. Finally, there was a type $\times$ task interaction, by items only $\left[F_{1}(1,30)=0.60, M S_{\mathrm{e}}=29,351\right.$, n.s., $F_{2}(1,62)=$ $\left.7.79, M S_{\mathrm{e}}=17,908\right]$. Planned comparisons of the type $\times$ task interaction by items revealed shorter RTs to shaded objects compared with silhouettes for both object decision and naming. The interaction arose because the difference between shaded objects and silhouettes was greater for object decision (106 msec) compared with naming $(67 \mathrm{msec})$.

(Manuscript received April 27, 2001;

revision accepted for publication December 19, 2001.) 